\title{
The erector spinae plane block: the case of the elusive mechanism of action
}

\author{
Jeff Gadsden, MD FRCPC FANZCA $D$
}

Received: 6 November 2020/Accepted: 7 November 2020/Published online: 6 January 2021

(C) Canadian Anesthesiologists' Society 2021

The mechanisms of nerve blockade should be easy to understand-local anesthetic is placed in the vicinity of a nerve, and the patient experiences a sensory block of the cutaneous, musculofascial, and osteal territory corresponding to that nerve. There is, thankfully, a direct and predictable relationship between administering local anesthetic at the brachial plexus or sciatic nerve and the patient experiencing a sensory loss in the hand or foot, respectively. Nevertheless, the erector spinae plane (ESP) block-a technique where local anesthetic is deposited deep to the erector spinae muscles to effect analgesia of the corresponding truncal area-appears to defy such a simple mechanistic explanation.

It is surprising that, for a block that has enjoyed a meteoric rise in popularity (at the time of writing, there are $>600$ unique PubMed records over the four years since its first description), we lack a unifying theory as to where and how that local anesthetic is acting after it is deposited. In this issue of the Journal, Drs Chin and El-Boghdadly present a comprehensive narrative review of the proposed mechanisms of the ESP block. ${ }^{1}$ The authors point out that while the block does seem to work (and for a surprisingly large number of clinical indications), we continue to lack a complete understanding of just how that local anesthetic is doing its job.

There are several aspects of the dynamics of this block that prevent us from accepting a unifying mechanism of action. First, there appears to be an inconsistent disposition of the local anesthetic after it is injected deep to the erector spinae muscles. Some cadaveric studies show that the

J. Gadsden, MD FRCPC FANZCA ( $\square)$

Division of Orthopaedic, Plastic and Regional Anesthesiology,

Duke University Medical Center, Durham, NC, USA

e-mail: jeff.gadsden@duke.edu injectate remains confined to the fascial envelope of the erector spinae muscle group itself. ${ }^{2,3}$ Indeed, in the most commonly accepted technique to perform the block, the block needle is advanced until contact is made with the dorsal aspect of the transverse process, to which the erector spinae muscle can be strongly adherent. It is all too easy to inadvertently perform a more superficial intramuscular injection, mistaking the elevation of muscle fibres within its fascial sheath for the elevation of the entire fascial plane. In these instances, the only nerves one could expect to be blocked are the more distal branches of the dorsal rami, assuming the local anesthetic remains in this muscular compartment. Other studies show penetration of dye injectate deep to the overlying erector spinae muscles, with staining of the surface of the external oblique muscle and/or ribs but little or no staining of deeper structures such as the paravertebral space, the ventral rami, or the intercostal nerves. ${ }^{4}$ With sufficient lateral extension along this plane, one might expect the injectate to reach the lateral cutaneous branches of the intercostal nerves (providing blockade of the lateral chest wall) but sparing the anterior and posterior midlines, much like a serratus plane block. There have been some reports of the sparing of the anterior cutaneous nerves of the thorax with the ESP block, suggesting that ventral rami are not consistently blocked. $^{5,6}$

Other dye studies show deeper spread through the costotransverse foramina and/or into the paravertebral space. ${ }^{7,8}$ The concept that the ESP block is a "backdoor" to the paravertebral space is certainly attractive. True paravertebral blockade can provide dense sensory blockade sufficient to perform awake mastectomy or herniorrhaphy, an outcome that has been elusive with other interfascial plane blocks that are better suited for only postoperative analgesia (e.g., transversus abdominis plane, serratus plane, 
pectoralis). Many anesthesiologists avoid performing paravertebral blocks because of the proximity to the pleura and epidural space and/or the risk of a bleeding into a deep compartment in anticoagulated patients. If the more superficial ESP block can deliver the same result as paravertebral block with less risk, ${ }^{9}$ then we may have achieved the trifecta in truncal blocks-i.e., simple to perform, safe, and effective. The problem lies in inconsistent spread to this space. For example, Chin and El-Boghdadly report that, of 123 individual thoracic ESP injections performed on cadavers, injectate was seen to spread into the paravertebral space in only 55 instances $(45 \%)$. The authors discuss the possibility of a diffusion gradient from the site of injection to the paravertebral space that simply may not be observable in cadaveric studies, as well as other dynamic factors (e.g., breathing, gliding fascial planes) that may promote spread to the paravertebral space in living humans. If an ESP block resulted in a reliable paravertebral block, one would expect both dorsal and ventral rami to be blocked, the result of which would be a cutaneous loss of sensation from the posterior midline around to the anterior midline. Nevertheless, clinical studies involving a thoracic ESP block suggest widely variable patterns of dermatomal effect to pinprick or cold sensation, some of which exhibit complete sparing of the anterolateral trunk. ${ }^{5,6}$ It is therefore surprising to read of the effectiveness of the ESP block in providing dense surgical blockade. For example, Malawat et al. performed ESP blocks using $25 \mathrm{~mL}$ of $0.5 \%$ bupivacaine in 30 patients undergoing modified radical mastectomy and reported complete surgical anesthesia in every patient. ${ }^{10}$ Any anesthesiologist who has performed multilevel paravertebral blocks for mastectomy, and endured the occasional incomplete block will agree that this is an enviable success story.

A second factor that adds to the ESP block mechanism mystery is the inconsistency in the relationship between volume of local anesthetic administered and the number of dermatomal segments affected. For example, a volume of $20 \mathrm{~mL}$ of local anesthetic appears to result in cutaneous blockade of as few as zero and as many as 13 dermatomal segments, ${ }^{11,12}$ with no obvious technical reason for the disparity. Cadaveric studies also show a large variation in craniocaudal spread with $20 \mathrm{~mL}$ of injectate ranging from three to 15 levels. ${ }^{2,3}$ It is likely that this relationship is influenced by some combination of factors relating to anatomy, gravity, injection pressure, timing of the assessment, and possibly other technical and/or pharmacologic causes.

Third, and perhaps most intriguing, is the finding that, despite instances of no clear cutaneous blockade, the ESP block still appears to provide a demonstrable postoperative analgesic effect, as evidenced by reductions in both reported pain scores and opioid consumption. Chin and El-Boghdadly discuss several possible mechanisms for this observation, including differential blockade of C-fibres, systemic local anesthetic absorption, a central neuraxial effect, as well as blockade of nociceptive fibres that are present in the thoracolumbar fascia itself. It is somewhat unsettling to perform a regional block and despite having the patient report no sensory loss, report good pain relief; perhaps the ongoing publication of positive outcomes make more tolerable the unknowns of why this is.

Chin and El-Boghdadly begin their review by quoting the fictional detective Sherlock Holmes, reflecting that when the impossible has been ruled out, the remaining explanation, however unlikely it may be, must represent the truth. On the surface, the ESP block seems impossibly marvellous-i.e., analgesia from the angle of the mandible down to the hip joint, easy to perform, and wonderfully forgiving of technical variance. That the local anesthetic may end up in any number of fascial compartments apparently seems irrelevant, and perhaps it is. Indeed, it is possible that these fascial planes we take such care to visualize are not all that "watertight" in the paraspinal region, and local anesthetic placed anywhere near the erector spinae muscle is sufficient to achieve good analgesic results. The very fact that these findings seem counterintuitive are exactly why we need to drill down on all the potential mechanisms. It is exciting that ESP block appears to be effective, but we are now at a point where we need to spend less effort on celebrating unique applications, and instead focus on rigorously clarifying how this block actually works through careful and patient investigation so that we can apply it more consistently in our practice. It is time to solve the ESP block riddle and render its mysteries "elementary".

\section{Le bloc du plan des muscles érecteurs du rachis : un mécanisme d'action insaisissable}

Les mécanismes des blocs nerveux devraient être faciles à comprendre - un anesthésique local est placé à proximité d'un nerf, puis le patient éprouve un bloc sensitif des territoires cutané, musculo-fascial et osseux correspondant à ce nerf. Il y a, heureusement, une relation directe et prévisible entre l'administration d'un anesthésique local au plexus brachial ou au nerf sciatique et le patient éprouvant une atteinte sensitive dans la main ou le pied, respectivement. Et pourtant, le bloc du plan des muscles érecteurs du rachis (ou bloc ESP), une technique 
où l'anesthésique local est déposé sous les muscles érecteurs du rachis pour réaliser l'analgésie de la zone tronculaire correspondante, semble défier une explication aussi simple.

Il est surprenant que, pour un bloc qui a connu une hausse fulgurante de popularité (au moment de la rédaction, plus de 600 articles uniques ont été publiés sur PubMed depuis la première description de ce bloc il y a quatre ans), nous ne disposions pas d'une théorie unificatrice décrivant l'endroit et la façon dont l'anesthésique local agit après son administration. Dans ce numéro du Journal, les Drs Chin et El-Boghdadly présentent un compte rendu narratif exhaustif des mécanismes suggérés du bloc ESP. ${ }^{1}$ Les auteurs soulignent qu'alors qu'il semble que le bloc fonctionne (et dans un nombre étonnamment élevé d'indications cliniques), nous ne comprenons toujours pas complètement comment l'anesthésique local agit.

Plusieurs aspects concernant la dynamique de ce bloc nous empêchent d'accepter qu'il possède un mécanisme d'action unique. Tout d'abord, il semble que la dispersion de l'anesthésique local après son injection postérieure aux muscles érecteurs du rachis ne soit pas constante. Certaines études cadavériques ont démontré que le produit injecté restait confiné à l'enveloppe fasciale même du groupe musculaire des érecteurs du rachis. ${ }^{2,3}$ En effet, dans la technique la plus communément utilisée pour réaliser le bloc, l'aiguille est dirigée jusqu'à ce qu'elle touche l'aspect dorsal du processus transverse, auquel les muscles érecteurs du rachis peuvent être fortement adhérents. Il est très facile de pratiquer, par inadvertance, une injection intramusculaire plus superficielle en prenant par erreur l'élévation des fibres musculaires dans leur gaine fasciale pour l'élévation du plan fascial dans son intégralité. Dans ces cas, les seuls nerfs que l'on pourrait s'attendre à anesthésier sont les branches plus distales des rameaux dorsaux, en supposant que l'anesthésique local reste dans ce compartiment musculaire. D'autres études ont montré une pénétration profonde du colorant injecté jusqu'aux muscles érecteurs du rachis sus-jacents, avec une coloration de la surface du muscle oblique externe et/ou des côtes, mais peu ou pas de coloration des structures plus profondes telles que l'espace paravertébral, les rameaux ventraux, ou les nerfs intercostaux. ${ }^{4}$ Avec une extension latérale suffisante le long de ce plan, on pourrait s'attendre à ce que le produit injecté atteigne les branches latérales cutanées des nerfs intercostaux (procurant un bloc de la paroi thoracique latérale) mais épargnant les lignes médianes antérieures et postérieures, un peu comme un bloc du plan du muscle serratus. Certains comptes rendus rapportent une préservation des nerfs cutanés antérieurs du thorax avec un bloc ESP, ce qui laisse penser que les rameaux ventraux ne sont pas uniformément bloqués. ${ }^{5,6}$
D'autres études avec colorant ont montré une diffusion plus profonde à travers les foramens costotransverses et/ou dans l'espace paravertébral. ${ }^{7,8}$ L'idée que le bloc ESP constitue une «porte d'entrée » pour l'espace paravertébral est certes séduisante. Un véritable bloc paravertébral peut procurer un bloc sensitif dense suffisant pour réaliser une mastectomie ou une cure d'hernie inguinale éveillée, un objectif jusqu'alors inatteignable avec les autres blocs du plan interfascial, mieux adaptés à l'analgésie postopératoire (par exemple, les blocs du plan abdominal transverse (TAP), du plan du muscle serratus, des pectoraux). De nombreux anesthésiologistes n'aiment pas réaliser des blocs paravertébraux en raison de leur proximité à la plèvre et à l'espace péridural et/ou du risque de saignement dans un compartiment profond chez les patients anticoagulés. Si le bloc ESP, qui est plus superficiel, peut offrir le même résultat qu'un bloc paravertébral avec moins de risque, ${ }^{9}$ nous avons peut-être remporté le tiercé gagnant des blocs du tronc : simple à exécuter, sécuritaire, et efficace. Le problème réside dans la dispersion inconstante dans cet espace. Par exemple, Chin et El-Boghdadly rapportent que, sur 123 injections individuelles d'ESP thoracique réalisées sur des cadavres, le produit injecté ne s'est propagé dans l'espace paravertébral que dans 55 cas $(45 \%)$ seulement. Les auteurs abordent la possibilité d'un gradient de diffusion du site d'injection vers l'espace paravertébral qui pourrait simplement ne pas être observable dans une étude cadavérique, ainsi que d'autres facteurs dynamiques (par exemple, la respiration, le glissement des plans fasciaux) qui pourraient favoriser la diffusion à l'espace paravertébral chez les sujets vivants. Si un bloc ESP produisait un bloc paravertébral fiable, on s'attendrait à ce que les rameaux dorsaux et ventraux soient bloqués, ce qui entraînerait une perte de sensation cutanée de la ligne médiane postérieure jusqu'à la ligne médiane antérieure. Cependant, les études cliniques examinant les blocs ESP thoraciques observent d'importantes variations dans l'effet sur le dermatome d'une piqûre d'épingle ou de la sensation de froid, et certains blocs affichent même une épargne complète du tronc antérolatéral. ${ }^{5,6} \mathrm{Il}$ est donc surprenant de lire des comptes rendus rapportant l'efficacité du bloc ESP pour procurer un bloc chirurgical dense. Par exemple, Malawat et coll. ont réalisé des blocs ESP en utilisant 25 $\mathrm{mL}$ de bupivacaïne $0,5 \%$ chez 30 patientes subissant une mastectomie radicale modifiée et rapportent une anesthésie chirurgicale complète pour chaque patiente. ${ }^{10}$ De tels résultats feraient rêver n'importe quel anesthésiologiste ayant réalisé des blocs paravertébraux à plusieurs niveaux pour une mastectomie, et ayant occasionnellement essuyé l'échec d'un bloc incomplet.

Un deuxième facteur qui ajoute au mystère entourant le mécanisme du bloc ESP a trait à l'inconstance de la 
relation entre le volume de l'anesthésique local administré et le nombre de segments dermatomaux affectés. Par exemple, un volume de $20 \mathrm{~mL}$ d'anesthésique local semble produire un bloc cutané affectant de zéro à 13 segments dermatomaux, ${ }^{11,12}$ sans raison technique évidente expliquant cette disparité. Les études cadavériques montrent également une grande variation dans la propagation craniocaudale avec $20 \mathrm{~mL}$ de produit injecté, allant de trois à 15 niveaux. ${ }^{2,3}$ Il est probable que cette relation soit influencée par une combinaison de facteurs liés à l'anatomie, à la gravité, à la pression d'injection, au moment de l'évaluation et peut-être à d'autres causes techniques et/ou pharmacologiques.

Troisièmement, et c'est peut-être là l'observation la plus intrigante : malgré les cas dans lesquels aucun bloc cutané clair n'est apparent, le bloc ESP semble tout de même procurer un effet analgésique postopératoire démontrable, comme en témoigne la réduction rapportée tant en matière de scores de douleur que de consommation d'opioïdes. Chin et El-Boghdadly présentent plusieurs mécanismes possibles pouvant élucider ce mystère, notamment un bloc différentiel des fibres $\mathrm{C}$, une absorption systémique de l'anesthésique local, un effet neuraxial central, ainsi qu'un bloc des fibres nociceptives présentes dans le fascia thoraco-lombaire. Il est quelque peu troublant de réaliser un bloc régional et que le patient ne signale aucune perte sensorielle tout en faisant état d'un bon soulagement de la douleur; la publication continue de résultats positifs rendra peut-être plus tolérable l'incompréhension de ces mécanismes.

Chin et El-Boghdadly commencent leur compte rendu en citant le détective de fiction Sherlock Holmes, selon lequel lorsque l'impossible a été exclu, l'explication restante, aussi improbable soit-elle, doit représenter la vérité. À première vue, le bloc ESP semble absolument merveilleux, c'est-à-dire une analgésie depuis la mandibule jusqu'à l'articulation de la hanche, facile à exécuter, et incroyablement indulgent en matière de variance technique. Il semble apparemment sans importance que l'anesthésique local puisse se retrouver dans toutes sortes de compartiments fasciaux; peut-être en est-il ainsi. En effet, il est possible que ces plans fasciaux que nous nous efforçons de visualiser avec beaucoup de soin ne sont pas si « imperméables » que cela dans la région paravertébrale, et qu'un anesthésique local placé n'importe où près des muscles érecteurs du rachis suffise à procurer une bonne analgésie. Le fait même que ces résultats semblent contreintuitifs est la raison pour laquelle nous devons approfondir tous les mécanismes potentiels. Il est certes formidable que le bloc ESP semble être efficace, mais nous atteignons maintenant une étape où il est moins important de célébrer ses applications particulières que de tenter de clarifier, de façon rigoureuse, la façon dont ce bloc agit véritablement.
Pour ce faire, nous devons mener des études avec minutie et patience. Ainsi, nous pourrons appliquer ce bloc de façon plus uniforme dans notre pratique. Il est temps de résoudre l'énigme du bloc ESP et de rendre ses mystères « élémentaires ».

Disclosures: None.

Funding statement: None.

Editorial responsibility: This submission was handled by Dr. Hilary P. Grocott, Editor-in-Chief, Canadian Journal of Anesthesia.

Déclaration: Aucune.

Déclaration de financement : Aucune.

Editorial responsibility: Cet article a été traité par Dr Hilary P. Grocott, rédacteur en chef, Journal canadien d'anesthésie.

\section{References}

1. Chin KJ, El-Boghdadly K. Mechanisms of action of the erector spinae plane (ESP) block: a narrative review. Can J Anesth 2021; this issue. https://doi.org/10.1007/s12630-020-01875-2

2. Aponte A, Sala-Blanch X, Prats-Galino A, Masdeu J, Moreno LA. Anatomical evaluation of the extent of spread in the erector spinae plane block: a cadaveric study. Can J Anesth 2019; 66: 886-93.

3. Harbell MW, Seamans DP, Koyyalamudi V, Kraus MB, Craner $R C$, Langley $N R$. Evaluating the extent of lumbar erector spinae plane block: an anatomical study. Reg Anesth Pain Med 2020; 45: 640-4.

4. Ivanusic J, Konishi $Y$, Barrington MJ. A cadaveric study investigating the mechanism of action of erector spinae blockade. Reg Anesth Pain Med 2018; 43: 567-71.

5. Zhang J, He Y, Wang $S$, et al. The erector spinae plane block causes only cutaneous sensory loss on ipsilateral posterior thorax: a prospective observational volunteer study. BMC Anesthesiol 2020; . https://doi.org/10.1186/s12871-020-01002-0.

6. Byrne $K$, Smith $C$. Human volunteer study examining the sensory changes of the thorax after an erector spinae plane block. Reg Anesth Pain Med 2020; 45: 761-2.

7. Yang HM, Choi YJ, Kwon HJ, Cho TH, Kim SH. Comparison of injectate spread and nerve involvement between retrolaminar and erector spinae plane blocks in the thoracic region: a cadaveric study. Anaesthesia 2018; 73: 1244-50.

8. Choi YJ, Kwon HJ, O J, et al. Influence of injectate volume on paravertebral spread in erector spinae plane block: an endoscopic and anatomical evaluation. PloS One 2019; https://doi.org/10. 1371/journal.pone.0224487.

9. Tsui BC, Kirkham $K$, Kwofie $M K$, et al. Practice advisory on the bleeding risks for peripheral nerve and interfascial blockade: rooted in evidence. Can J Anesth 2020; 67: 379-80.

10. Malawat A, Verma $K$, Jethava D, Jethava DD. Erector spinae plane block for complete surgical anaesthesia and postoperative analgesia for breast surgeries: a prospective feasibility study of 30 cases. Indian J Anaesth 2020; . https://doi.org/10.4103/ija.IJA_ 639_19.

11. Fang B, Wang $Z$, Huang $X$. Ultrasound-guided preoperative single-dose erector spinae plane block provides comparable 
analgesia to thoracic paravertebral block following thoracotomy: a single center randomized controlled double-blind study. Ann Transl Med 2019; . https://doi.org/10.21037/atm.2019.03.53.

12. Barrios A, Camelo J, Gómez J, et al. Evaluation of sensory mapping of erector spinae plane block. Pain Physician 2020; 23: E289-96.
Publisher's Note Springer Nature remains neutral with regard to jurisdictional claims in published maps and institutional affiliations. 